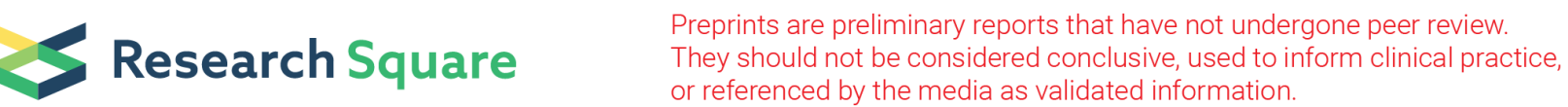

\section{Use of miR-34a, miR-126, and miR-125a-5p expression level as diagnostic factor and therapeutic target for $\alpha$-L-guluronic acid and $\beta$-D- mannuronic acid in Type-I diabetic rat models}

Mohammad sharifzade

Tehran University of Medical Sciences

Mohammad reza emami ( $\sim$ emami.mohammadreza70@gmail.com )

Tehran University of Medical Sciences https://orcid.org/0000-0001-5911-2654

Aghil Esmaeili-Bandboni

Tehran University of Medical Sciences

Mohammad Hassan Javanbakht

Tehran University of Medical Sciences

Abbas Mirshafiey

Tehran University of Medical Sciences

\section{Research}

Keywords: MicroRNAs, Diabetes, a-L-guluronic acid, $\beta$-D-mannuronic acid

Posted Date: February 28th, 2020

DOI: https://doi.org/10.21203/rs.2.24834/v1

License: (c) (i) This work is licensed under a Creative Commons Attribution 4.0 International License.

Read Full License 


\section{Abstract}

Type 2 diabetes mellitus (T2DM) affects microRNAs (miRNAs) expression that are known as Posttranscriptional repressors of gene expression in plants and animals. This research aimed to investigate the expression levels of miR-34a, miR-126, miR-125a-5p, and miR-29b for diabetes diagnosis, and the effect of a-L-guluronic acid and $\beta$-D-mannuronic acid supplementation on the expression pattern of these miRNAs in diabetic rats. Total miRNAs were extracted and evaluated by qRT-PCR method. body weight, fasting insulin levels, food intake and Vitamin D level in four group after 4-week intervention also measured. The analyzes showed significant increase in expression levels of miR-126 and miR-125a-5p, in diabetic control groups $(p=0.001)$. Also, miR-29b was undetectable in the sera of experimental rats. After treatment with $\beta$-D-mannuronic acid, the expression level of miR-34a was upregulated in diabetic rats $(p=0.026)$, but $a-L$-guluronic acid was not significantly effective. Also, expression levels of miR-126 $(p=0.026)$ and miR-125a-5p ( $p=0.041)$ were downregulated significantly in diabetic rats, after treatment with $\beta$-D-mannuronic acid and, $a$-L-guluronic acid respectively. these results suggest that use of miR 126 ,125a-5p and miR 34a for diagnosing diabetes is sensible, also we can consider $\beta$-D-mannuronic acid as new therapeutic agent for correct disturbance in miRNA profile of diabetic rats.

\section{Introduction}

Diabetes mellitus (DM) is a metabolic disorder characterized by hyperglycemia with disturbances in carbohydrate, fat, and protein metabolism due to a defect in insulin action and secretion (1). Major risk factor for cardiovascular disease exists in subjects with a history of Type 2 diabetes and most patients with diabetes die from complications of atherosclerosis (2). Oxidized low density lipoprotein (ox-LDL) plays a major role in the start and progression of atherosclerosis, in a diabetes patient serum ox-LDL level increase with the length of diabetes, even though the patients LDL-cholesterol level is maintained in the normal range, ox-LDL produced from LDL in an oxidative stress situation (3). The previous study approved that $0 x-L D L$ accelerates atherosclerotic plaque formation and may be contributing to the exacerbate of microvascular complications in diabetes (4). Mir-125a-5p was found to interfere lipid uptake and to decrease the secretion of some inflammatory cytokines (interleukin-6, interleukin-2, tumor necrosis factor- $a$, transforming growth factor-beta) in ox-LDL-stimulated monocyte(5). in diabetic subject mir125a-5p upregulate by ox-LDL and inflammatory cytokine as a protect factor and interfere with their action(5, 6).

As mentioned above, one of the causes of death of a diabetic patient is atherosclerosis, which is exacerbated by ox-LDL. Alginate is commonly extracted from brown algae. Its chain is made of homopolymeric regions of $\beta$-D-mannuronic acid and $a-L$-guluronic acid. The previous study approved that Na-alginate (at $600 \mathrm{mg} \cdot \mathrm{kg}^{-1}$ ) increased weight of diabetic rat significantly comparing to normal control, and decreases pre-prandial and postprandial glucose (7). Another study indicated that a-L-guluronic acid had immunomodulatory effect by decrease the expression of Toll-like receptor 2 (TLR2) and Nuclear Factor-kB (NF-kB)genes in Common variable immunodeficiency (CVID) patients (8). The $\beta$-D-mannuronic acid as a novel herbal extract drug plays an important role in the management of inflammatory 
diseases(9). $\beta$-D-mannuronic acid molecule is an agent with the lowest molecular weight and has no toxicity compared with other anti-inflammatory drug (10).

MiRNAs are short endogenous RNAs (about 19-24 nucleotides) that repress post-transcriptionally gene expression in eukaryotes. There is evidence suggesting that miRNAs are involved in many of pathological processes (11). A recent paper has approved that the expression of microRNAs profiles change in diabetes (12). The previous studies demonstrate that miRNAs are present in the serum and plasma of rats and other animals such as mice, humans, The levels of miRNAs in serum are reproducible, and stable among individuals of the same species (13). Up-regulation of miR-29b in cell line induced by ox-LDL involved in reducing the methylation of DNA and the migration of the primary human aortic smooth muscle cell (HASMC) which mediated epigenetic regulation, maybe considers as a novel mechanism in atherosclerosis (14). MiR-34a could induce apoptosis by inducing G0/G1 arrest in mouse pancreatic beta cells.

In addition, higher expression of miR-34a may be related to the pathogenesis of T2DM. Then the serum level of miR-34a is a potential biomarker for T2DM diagnosis. Moreover, the expression of mi-RNA34a gene increases in human atherosclerosis plaque $(15,16)$. Macrophages, absorbed into the adipose tissue through C-C motif chemokine receptor-2 (CCR2), were postulated play a role in the development of insulin resistance in type 2 diabetes mellitus (17). In another previous study, upregulation of miR-125a-5p was observed in regulatory $T$ (Treg) cells extracted from pancreatic draining lymph nodes (PLN) of T1D patients. TNFR2 (Tumor necrosis factor receptor 2) and C-C Chemokine Receptor type-2 (CCR2) were identified as miR-125a-5p target genes. For instance, Increase expression of miR-125a-5p in Treg cells of diabetes patients which result in the reduced expression of CCR2, thus limiting their migration and function in the pancreas (18). Also, previous studies showed that miR-126 expression level decreased in T2DM manifesting. It suggests reduced miR-126 is a predictor for the onset of T2DM. Furthermore, the miR-126 level acts as an essential factor in the efferocytosis process of apoptotic myocytes by macrophages, in high glucose conditions (HG) such as diabetes $(19,20)$.

Due to considering the high prevalence of diabetes and its complication in Iran and the world, as well as the lack of adequate studies on the effects of a-L-guluronic acid and $\beta$-D-mannuronic acid on serum levels of miRNAs, the present study conducted in the animal model of type I diabetes on Sprague-Dawley rats to determine the effectiveness of $a$-L-guluronic acid and $\beta$-D-mannuronic acid on four miRNAs (miR34a, miR-126, miR-125a-5p, and miR-29b) expression level.

\section{Materials And Methods}

\section{Diabetic Rat Model}

Twenty-one Young Adult Sprague-Dawley rats (180-260 g) were used for this interventional study. Before the start of the experiments in order to adaptation, the animals were housed under suitable conditions of temperature, and light for two days and feed with standard rat chow diet in a 12:12-h light-dark cycle. This study was performed according to the animal care protocol, approved by the animal care and 
research committee of Tehran University of Medical Sciences. The experimental model of type 1 DM (insulin-dependent DM) was induced by a single intraperitoneal injection of $60 \mathrm{mg} \mathrm{STZ/kg} \mathrm{(Sigma,} \mathrm{St.}$ Louis, MO) in $1 \mathrm{mM}$ phosphate buffer. After $48 \mathrm{~h}$ of STZ injection, the development of DM was confirmed with the animal's fasting blood sugar measurement (using a glucometer set). Fasting serum glucose was considered as an index of DM development (glucose level $>200 \mathrm{mg} / \mathrm{dL}$ ). Rats became hyperglycemic at two days after STZ injection and maintained hyperglycemic before being killed.

\section{Intervention}

\section{Experimental procedure}

The rats were divided into four groups, 6 animals in each group as follow.

Group I Healthy control rats was administered normal saline $0.5 \mathrm{~mL}$

Group II STZ-induced diabetic control rats was given vehicle flaxen oil $0.5 \mathrm{~mL}$

Group III Diabetic rats were given a-L-guluronic acid $(25 \mathrm{mg} / \mathrm{kg}$ ) (manufactured by Sigma Corporation of America SIGMA R2625) in $0.5 \mathrm{ml}$ Flaxen oil solution using an intraperitoneal injection for 30 days. At the first three days, injections were performed one time per day. Other injections were administered everyother-days.

Group IV Diabetic rats were given $\beta$-D-mannuronic (manufactured by Sigma Corporation of America SIGMA R2625) $(25 \mathrm{mg} / \mathrm{kg})$ in $0.5 \mathrm{ml}$ Flaxen oil solution using an intraperitoneal injection for 30 days. At the first three days, injections were performed one time per day. Other injections were administered everyother-days.

\section{Blood samples:}

At the end of the study, after weighing and measuring blood glucose, rats were anesthetized with Ketamine and Xylazine and then sacrificed with chloroform. A peripheral blood specimen was drawn from each rat ( $6 \mathrm{~mL} / \mathrm{rat})$. EDTA was used as an anticoagulant. Plasma was separated by centrifugation $\left(1200 \times \mathrm{g}\right.$ for $10 \mathrm{~min}$ at $\left.4^{\circ} \mathrm{C}\right)$ and stored in RNase-free tubes $(1.5 \mathrm{~mL})$ at $-80^{\circ} \mathrm{C}$.

\section{miRNA extraction}

MiRNAs were extracted from $400 \mathrm{ml}$ of plasma using Hybrid-R miRNA isolation Kit (GeneAll, South Korea) according to manufacturer's protocol. Concentration and purity of extracted miRNA were assessed by Thermo Scientific ${ }^{\text {TM }}$ NanoDrop ${ }^{\mathrm{TM}}$ 2000/2000c Spectrophotometer.

\section{Primers}

Revers Transcription (RT) primer: First strand of cDNA was synthesized from miRNA templates using a stem loop cDNA synthesis primer, as described by Kramer (21). 
Reverse primer: Universal reverse primer was obtained from Kramer article (21).

Forward Primers: Forward primers for each miRNA were designed according to the Kramer (21) and Busk (22) articles. Sequences of all forward primer (miR-34a, miR-126, miR-125a-5p, miR-29b, and miR-16 as normalizing factor) were shown in Table 1. All the primers were ordered for synthesis in Bioneer company, Daejeon, Republic of Korea.

\section{CDNA synthesis and Real-time quantitative RT-PCR}

CDNAs were synthesized from $5 \mathrm{ul}$ of total miRNA using PrimeScript ${ }^{\circledR}$ 1st Strand cDNA Synthesis Kit (Takara Bio, Siga, Japan, cat number: \#6110A) according to the manufacturer's protocol. The expression of miRNAs in treated and control groups was detected by using SYBRGreen PCR master mix (Takara Bio, Siga, Japan, cat number: \#RR820L). The reaction was performed in a real-time quantitative PCR machine (Applied Biosystem step one plus). Real time PCR reactions were carried out using the first chain of cDNA as the pattern. Each reaction contained $2 \mu \mathrm{L}$ of cDNA, $10 \mu \mathrm{L}$ of SYBR Green I Master mix, $1 \mu \mathrm{L}$ of miRNA-specific primers, $1 \mu \mathrm{L}$ of upstream universal primer, $5.5 \mu \mathrm{l} \mathrm{H}_{2} \mathrm{O}$, and $0.5 \mu \mathrm{L}$ Rox. A mean of triplicate findings was used as the result for every sample. MiR-16 was used as an endogenous reference for normalization. Relative changes in gene expression were calculated by $2-\Delta \Delta \mathrm{Ct}$, the definition of is $\triangle \triangle \mathrm{Ct}=$ (Ct miRNAs - Ct miR-16) of the treatment group - (Ct miRNAs - Ct miR-16) mean of the control group.

\section{Insulin, and Glucose assay}

The glucose level was measured by glucose oxidase protocol and using glucose kit of the (biochemistry, Tehran, cat number: 117500). Insulin levels were measured using (DiaMetra, Perugia Italy, cat number: DKO076) ELISA kit.

\section{Statistical analysis}

Data were analyzed using Kolmogorov-Smirnov test. Due to non-normal distribution of data on gene expression and final food intake, non-parametric methods were used to analyze these variables. KruskalWallis test and one-way ANOVA were used for between group analysis of these variables. Since data on fasting blood glucose, fasting insulin levels and weight were normal distribution one-way ANOVA test with LSD post Hoc was used to compare groups. $(P<0.05)$ was considered as the level of significance. Kruskal-Wallis test was used to compare the mean concentration of different microRNAs in the control and intervention groups. SPSS 22.0 statistical software was used for statistical analysis and Graph Pad Prism V 6.3 was used for graph drawing. We used the Kruskal-Wallis test for comparison between multiple groups. $P<0.05$ represent a statistical significance.

Table 1. Sequences of primers 


\begin{tabular}{ccl}
\hline Primer name & Accession number of miRs ${ }^{\text {a }}$ & \multicolumn{1}{c}{ Sequences } \\
\hline Stem loop RT primer & - & GTCGTATCCAGTGCAGGGTCCGAGGTATTCGCACTGGATACGACNNNNNN \\
\hline Revers primer & - & CCAGTGCAGGGTCCGAGGTA \\
\hline F-miR-34a & MI0000584 & GGGGTGGCAGTGTCTTAGCT \\
\hline F- miR-29b & MI0000143 & GGGGGGTAGCACCATTTGAAATC \\
\hline F- miR-125a-5p & MIMAT0000135 & GGGGTCCCTGAGACCCTTTAAC \\
\hline F- miR-126a & MI0000153 & GGGGGTCGTACCGTGAGTAAT \\
\hline F- miR-16 & MI0000565 & GGGGGGTAGCAGCACGTAAATA \\
\hline
\end{tabular}

RT; Reverse Transcription, F; forward primer,

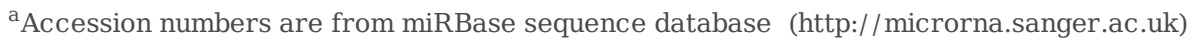

\section{Result}

Body and biochemical characteristics at baseline and after the interventions in healthy diabetic rats showed in Table 2. Body weight of Diabetic group treat with a-L-guluronic acid increased significantly compare to diabetic control (DC), but blood glucose did not decrease in DC rats after treatment. The results of one-way ANOVA test showed no significant difference in the initial weight $(P=0.879)$ and initial Food intake $(p=0.633)$ between the studied groups, but there was a significant difference in the final weight $(P=0 / 031)$ and the final food intake $(P=0 / 005)$. 
Table 2

Body and biochemical characteristics in Healthy and Diabetics control groups at baseline and after the interventions with $\mathrm{a}-\mathrm{L}$-guluronic acid and $ß-\mathrm{d}$-mannuronic acid.

\begin{tabular}{|llllll|}
\hline Variable & Group & & & & P-value \\
\hline & $\begin{array}{l}\text { a-L-guluronic } \\
\text { acid( }(\mathrm{n}=6)\end{array}$ & $\begin{array}{l}\text { Diabetic rats B-d- } \\
\text { mannuronic acid }(\mathrm{n}=4)\end{array}$ & $\begin{array}{l}\mathrm{DC}(\mathrm{n}) \\
=6)\end{array}$ & $\begin{array}{l}\mathrm{HC}(\mathrm{n}) \\
=5)\end{array}$ & $\begin{array}{l}\text { Between } \\
\text { groups }\end{array}$ \\
\hline $\begin{array}{l}\text { Weight before } \\
\text { intervention (gr) }\end{array}$ & $232 \pm 37$ & $244 \pm 45$ & $\begin{array}{l}231 \pm \\
45\end{array}$ & $\begin{array}{l}223 \\
\pm 18\end{array}$ & $0 / 879$ \\
\hline Final weight (gr) & $337 \pm 105^{\mathrm{a}}$ & $272 \pm 61$ & $208 \pm$ & 303 & $0 / 039$ \\
\hline $\begin{array}{l}\text { Food intake before } \\
\text { intervention (gr) }\end{array}$ & $21 \pm 1$ & $23 \pm 1$ & 41 & \pm 32 & \\
\hline $\begin{array}{l}\text { Final food intake (gr) } \\
\text { Fasting blood sugar }\end{array}$ & $26 \pm 1$ & $25 \pm 1$ & $24 \pm 5$ & $23 \pm$ & $0 / 633$ \\
\hline $\begin{array}{l}\text { Fefore } \\
\text { (mg/dl) }\end{array}$ & $261 \pm 86^{\mathrm{b}}$ & $264 \pm 47^{\mathrm{b}}$ & $23 \pm 3$ & $30 \pm$ & $0 / 005$ \\
\hline $\begin{array}{l}\text { Fasting blood sugar } \\
\text { final } \\
\text { (mg/dl) }\end{array}$ & $241 \pm 145$ & $330 \pm 115^{\mathrm{c}}$ & $300 \pm$ & $96 \pm$ & $0 / 001$ \\
\hline $\begin{array}{l}\text { Final fasting insulin } \\
\text { ( } \mu \text { lU/ml) }\end{array}$ & $5 \pm 1^{\mathrm{a}}$ & $6 / 095^{\mathrm{a}}$ & $86^{\mathrm{b}}$ & 14 & \\
\hline
\end{tabular}

DC; Diabetic Control, HC; Healthy Control

a; The mean values are statistically significantly higher than the diabetic control group ( $P \llbracket 0.05)$.

b; The mean values are statistically significantly higher in comparison with the healthy control group $(P \otimes 0.05)$.

c; The mean of the values is statistically significantly higher than the healthy control group (Pख0.05).

To investigated the differences of miRNAs between diabetic and non-diabetic groups, four miRNAs related to diabetes were measured in the sera of experimental rat using qRT-PCR after four-week intervention. Kruskal Wallis test was used for analysis. This analysis showed differences in the expression levels of miR-126, miR-125a-5p and miR-34a, in sera between groups (healthy, and diabetic control groups, and two diabetic groups treated with a-L-guluronic acid and $\beta$-D-mannuronic acid. MiR$29 \mathrm{~b}$ was undetectable in our rats. MiR-34a expression level was lower significantly in the diabetic group

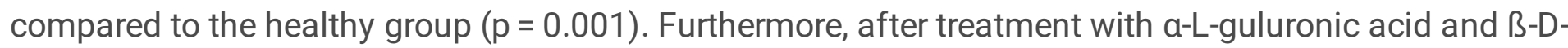
mannuronic acid supplementation in diabetic rats, the expression level of miR-34a increased. Increase the level of miR-34a after treatment was significantly in B-D-mannuronic acid group $(p=0.026)$, but was not

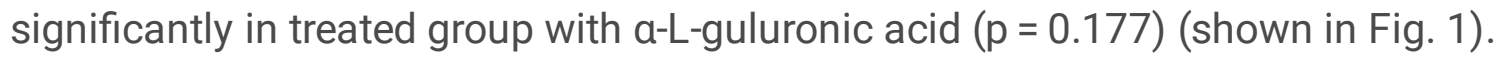


MiR-126 significantly increased in the diabetic group compared to healthy control $(p=0.001)$. After treatment with a-L-guluronic acid, and B-D-mannuronic acid supplementation mir126 expression downregulated. This change was significant in B-D-mannuronic acid treatment group $(p=0.026)$, and was not significant in the a-L-guluronic acid treatment group $(p=0.177)$ (Show in Fig. 2$)$. Also, the miR$125 a-5 p$ increase in the diabetic group compared to the healthy control group $(p=0.001)$. This change was decreased after treatment with a-L-guluronic acid, D-mannuronic supplementation. Fold change caused by $a$-L-guluronic acid was significant $(p=0.041)$, but caused by ß-D-mannuronic acid treatment was not significant $(p=0.124)$ (shown in Fig. 3).

\section{Discussion}

It was seen that epigenetic and genetic changes could involve in diabetes and progression of insulin resistant(23). Several studies revealed the involvement of miRNAs in key cellular activities including proliferation, differentiation, and metabolic integration and some disease like diabetes (24). Some websites like miRBase present about hundreds of human miRNAs. For the Selection suitable miRNAs for our study, we reviewed the relevant paper and selected the four miRNAs, which have been proven engage in T2DM. The major finding of our study is that miR-34a downregulate, and miR- $125 a-5 p$, and miR-126 increased significantly in the diabetic rats compare to the healthy rats. Furthermore, about these three miRNAs, after treatment with one supplement (B-D-mannuronic acid) significantly improved diabetes in rats, but after treatment with another selective supplement ( $\mathrm{a}-\mathrm{L}$-guluronic acid) improved diabetes in rats very little and not significantly. Also, we could not detect miR-29b in rats. This result confirm Zhang et al. (25), finding that miR-29b in the serum of rat is undetectable.

According to the report of Jahromi et al. (26), $\beta$-D-mannuronic acid is a novel immunosuppressive drug and able to modify toll-like receptors (TLR) signaling by suppressing the NF-KB and miR-146a as a new therapeutic approach. The previous study revealed that miR-34a may be related to the pathogenesis of T2DM, Liu et al. (15), suggested that miR-34a can induce apoptosis by inducing G0/G1 arrest in mouse pancreatic beta cells. Also, Kong et al. (27), have shown that in patients with a history of diabetes serum miR-34a was significantly upregulated compared to nondiabetics patients, but contrary to our prediction, in our study miR-34a expression decrease in DC Compared to $\mathrm{HC}$ (Healthy control). In this study, we showed that $\beta$-D-mannuronic acid could change miR-34a expression significantly but a-L-guluronic acid could not change miR-34a expression.

The previous study approved that Serum miR-126 was significantly lower in impaired glucose tolerance (IGT), impaired fasting glucose (IFG) subjects and T2DM patients than in healthy controls (20). Serum miR-126 was increased significantly in IGT-IFG and T2DM patients by diet therapy, insulin, and exercise for six months. miR-126 is highly enriched in endothelial cells and contributes to the repair and maintenance of angiogenesis, vascular integrity $(28,29)$. Our results in contrast to the findings of previous studies showed that miR-126 in plasma of DC significantly upregulated compared to HC group, which probably reduction of mir126 involved in the pathogenesis and complication of diabetes (30). Also after treatment with $\beta$-D-mannuronic acid, the expression level of miR-126 decreased significantly 
compared to the diabetic control group that did not receive the treatment. This inconsistency may be due to differences in study time, Previous studies have included subject with a long history of diabetes and hyperglycemia, which may reduce the expression of mir126 and thus expose people to cardiovascular disease (29), while our study was designed over a one-month period mir126, which is considered a protective factor, may increase in the short term.

MiR-125a-5p regulated the expression level of genes in the mitogen-activated protein kinase (MAPK) signaling pathway that is important in T2DM pathogenesis. Ciccacci et al.(31), and Herrera et al.(32), showed that the level of this microRNA increased in human and rat with T2DM disease compare to the healthy individuals, respectively $(31,32)$. Our results confirm the findings of these previous studies and reveal that miR-125a-5p significantly increases in DC compared to HC. Also, after treatment with a-Lguluronic acid, the expression level of miR-125a-5p decreased significantly compared to the diabetic control group that did not receive the treatment.

As observed in the previous study, alginate could decrease fasting plasma glucose in diabetic rats(7). Our study, in consistency with previous studies, has shown that supplementation with using a-L-guluronic acid and $\beta$-D-mannuronic acid can decrease fasting blood glucose but in controversy with previous study this effect it's not significant In this study, we confirm the previous finding that the body weight of diabetic rats increased after intervention with a-L-guluronic acid, ß-D-mannuronic acid supplementation reverse mir-34a downregulated by diabetes and downregulated mir126 which increased by diabetes and also a-L-guluronic acid decrease mir125a-5p that increased by diabetes. Therefore, B-D-mannuronic acid seems to be better therapeutic factor than a-L-guluronic acid to regulate the expression level of miRs and their target genes. Although these two factors increase fasting insulin significantly compare to DC, help to gain weight losted in diabetes and increase gene expression of mir125a-5p which may because of modulate inflammatory cytokine. B-D-mannuronic acid can hope the more relevant cure in the future by co-treatment with other therapeutic agents. a miRNA regulates many mRNAs, and conversely, a mRNA is regulated by several miRNAs. it's reasonable to measure mRNA also and inflammatory cytokine but because budget restriction we couldn't do it and its biggest limitation for our study. Furthermore, provide a precise and early diagnosis of diabetes to delay progression and early intervention for minimizing complication is necessary. Complex etiology, which involves, genetics, diet, and environmental factors, is as diagnostic and therapeutic challenges for DM. Currently, physician and health staff are using traditional methods include levels of $\mathrm{HbA} 1 \mathrm{c}$ and glucose; However, these biomarkers are not early diagnostic factors for DM(33). Hence, the discovery of the new therapeutic agent and biomarkers is necessary. In this article, we evaluated three microRNAs as the early diagnostic factors for DM.

\section{Conclusion}

a-L-guluronic acid and $\beta$-D-mannuronic acid increased fasting insulin, help to gain weight losted in diabetes and increase gene expression of mir125a-5p as a protective agent which may because of modulate inflammatory cytokine. ß-D-mannuronic acid can hope the more relevant effect in the future by 
co-treatment with other therapeutic agents. Therefore, we can suggest ß-D-mannuronic acid as new therapeutic agent which need further researches and also miRs as a diagnosing diabetes factor.

\section{Abbreviations}

MiRNAs

MicroRNAs

DM

Diabetes mellitus

Ox-LDL

Oxidized low density lipoprotein

CVID

Common variable immunodeficiency

TLR2

Toll-like receptor 2

NF-kB

Nuclear Factor-kB

HASMC

Human aortic smooth muscle cell

T2DM

Type 2 diabetes mellitus

CCR2

Chemokine receptor-2

PLN

Pancreatic draining lymph nodes

T1D

Type 1 diabetes

CCR2

C-C Chemokine Receptor type-2

$\mathrm{HC}$

Healthy control

DC

Diabetic control

RT

Revers Transcription

(IGT)

Impaired glucose tolerance

(IFG)

Impaired fasting glucose 


\section{Declarations}

Acknowledgments

The authors acknowledge grant supports from Tehran University of Medical Sciences and Health Services (T.U.M.S.).

\section{Authors' contributions}

Mohammad sharifzade (MS) and Mohammad Hassan Javanbakht (MJ)conceived and designed the experiments. Mohammad sharifzade (MS) and Mohammad Hassan Javanbakht (MJ) performed the experiments. MS carried out all the procedures that involved experimental animals. MS, ME (Mohammad reza emami), (Abbas Mirshafiey) AM and AB (Aghil Esmaeili-Bandboni) analyzed the data. All authors read and approved the final manuscript.

\section{Funding}

This research was supported by grant kindly provided by Tehran University of Medical Sciences \& Health Services.

\section{Availability of data and materials}

Data are all contained within the article. Additional analysis relative to the experimental model were previously published in Mortazavi-Jahromi SS, Alizadeh S, Javanbakht MH et al (2018a) Anti-diabetic effect of $\beta$-D-mannuronic acid (M2000) as a novel NSAID with immunosuppressive property on insulin production, blood glucose, and inflammatory markers in the experimental diabetes model. Arch Physiol Biochem. https://doi.org/10.1080/13813455.2018.1481094

\section{Ethics declarations}

\section{Ethics approval and consent to participate}

All animal's procedures were undertaken according to the Iranian ethical guidelines for the use of experimental animals. http://ethics.research.ac.ir/IR.TUMS.VCR.REC.1397.1119

\section{Consent for publication}

All authors approved the final version of this manuscript.

\section{Competing interests}


The authors declare that they have no competing interests.

\section{References}

1. Association AD. 2. Classification and diagnosis of diabetes. Diabetes care. 2016;39(Supplement 1):S13-S22.

2. Zinman B, Wanner C, Lachin JM, Fitchett D, Bluhmki E, Hantel S, et al. Empagliflozin, cardiovascular outcomes, and mortality in type 2 diabetes. New England Journal of Medicine. 2015;373(22):211728.

3. Nakhjavani M, Khalilzadeh O, Khajeali L, Esteghamati A, Morteza A, Jamali A, et al. Serum oxidizedLDL is associated with diabetes duration independent of maintaining optimized levels of LDLcholesterol. Lipids. 2010;45(4):321-7.

4. Wegner M, Piorunska-Stolzmann M, Araszkiewicz A, Zozulinska-Ziolkiewicz D, Naskret D, Uruska A, et al. Does oxidized LDL contribute to atherosclerotic plaque formation and microvascular complications in patients with type 1 diabetes? Clinical biochemistry. 2012;45(18):1620-3.

5. Chen T, Huang Z, Wang L, Wang Y, Wu F, Meng S, et al. MicroRNA-125a-5p partly regulates the inflammatory response, lipid uptake, and ORP9 expression in oxLDL-stimulated monocyte/macrophages. Cardiovascular research. 2009;83(1):131-9.

6. Böhm F, Pernow J. The importance of endothelin-1 for vascular dysfunction in cardiovascular disease. Cardiovascular research. 2007;76(1):8-18.

7. Husni A, Pawestri S, Isnansetyo A. Blood glucose level and lipid profile of alloxan-induced diabetic rats treated with $\mathrm{Na}$-alginate from seaweed Turbinaria ornata (Turner) J. Agardh. Jurnal Teknologi. 2016;78(4-2).

8. Farhang H, Sharifi L, Dallal MMS, Moshiri M, Norouzbabaie Z, Bokaie S, et al. The Immunomodulatory Role of G2013 (a-L-Guluronic Acid) on the Expression of TLR2 and TLR4 in HT29 cell line. Current Drug Discovery Technologies. 2019;16(1):91-5.

9. Kashefi S, Omranipour R, Mahmoodzadeh H, Ahmadi H, Mirshafiey A. Clinical improvement of diabetes mellitus type 1 by bD-mannuronic acid (M2000) in a breast cancer patient-as a case report. Clinical Diabetology. 2019;8(4):227-9.

10. Fattahi MJ, Abdollahi M, Agha Mohammadi A, Rastkari N, Khorasani R, Ahmadi $\mathrm{H}$, et al. Preclinical assessment of $\beta$-d-mannuronic acid (M2000) as a non-steroidal anti-inflammatory drug. Immunopharmacology and immunotoxicology. 2015;37(6):535-40.

11. Esmaeili-Bandboni A, Bagheri J, Bakhshandeh AR, Mohammadnejad J, Sadroddiny E, editors. Serum Levels of miR-155, miR-326, and miR-133b as Early Diagnostic Biomarkers for the Detection of Human Acute Heart Allograft Rejection in Comparison with Serum Cardiac Troponin T. The heart surgery forum; 2018.

12. Qin B, Xiao B, Liang D, Xia J, Li Y, Yang H. MicroRNAs expression in ox-LDL treated HUVECs: MiR-365 modulates apoptosis and Bcl-2 expression. Biochemical and biophysical research communications. 
2011;410(1):127-33.

13. Chen X, Ba Y, Ma L, Cai X, Yin Y, Wang K, et al. Characterization of microRNAs in serum: a novel class of biomarkers for diagnosis of cancer and other diseases. Cell research. 2008;18(10):997.

14. Chen K-C, Wang Y-S, Hu C-Y, Chang W-C, Liao Y-C, Dai C-Y, et al. OxLDL up-regulates microRNA-29b, leading to epigenetic modifications of MMP-2/MMP-9 genes: a novel mechanism for cardiovascular diseases. The FASEB Journal. 2011;25(5):1718-28.

15. Liu H, Jin M, Tian H, Li J, Li N, Yan S. MiR-34a and miR-34C are involved in the pathogenesis of type2 diabetes by modulating the cell cycle of pancreatic beta-cell. INTERNATIONAL JOURNAL OF CLINICAL AND EXPERIMENTAL PATHOLOGY. 2016;9(5):5340-5.

16. Raitoharju E, Lyytikäinen L-P, Levula M, Oksala N, Mennander A, Tarkka M, et al. miR-21, miR-210, miR-34a, and miR-146a/b are up-regulated in human atherosclerotic plaques in the Tampere Vascular Study. Atherosclerosis. 2011;219(1):211-7.

17. Di Prospero N, Artis E, Andrade-Gordon P, Johnson D, Vaccaro N, Xi L, et al. CCR2 antagonism in patients with type 2 diabetes mellitus: a randomized, placebo-controlled study. Diabetes, Obesity and Metabolism. 2014;16(11):1055-64.

18. Sebastiani G, Ventriglia G, Stabilini A, Socci C, Morsiani C, Laurenzi A, et al. Regulatory T-cells from pancreatic lymphnodes of patients with type-1 diabetes express increased levels of microRNA miR125a-5p that limits CCR2 expression. Scientific reports. 2017;7(1):6897.

19. Babu SS, Thandavarayan RA, Joladarashi D, Jeyabal P, Krishnamurthy S, Bhimaraj A, et al. Microrna126 Overexpression Rescues Impaired Efferocytosis in Diabetes. Am Heart Assoc; 2016.

20. Liu Y, Gao G, Yang C, Zhou K, Shen B, Liang H, et al. The role of circulating microRNA-126 (miR-126): a novel biomarker for screening prediabetes and newly diagnosed type 2 diabetes mellitus. International journal of molecular sciences. 2014;15(6):10567-77.

21. Kramer MF. Stem-loop RT-qPCR for miRNAs. Current protocols in molecular biology. 2011;95(1):15.0. 1-.0. .

22. Busk PK. A tool for design of primers for microRNA-specific quantitative RT-qPCR. BMC bioinformatics. 2014;15(1):29.

23. Patti ME, Butte AJ, Crunkhorn S, Cusi K, Berria R, Kashyap S, et al. Coordinated reduction of genes of oxidative metabolism in humans with insulin resistance and diabetes: Potential role of PGC1 and NRF1. Proceedings of the National Academy of Sciences. 2003;100(14):8466-71.

24. Stefani G, Slack FJ. Small non-coding RNAs in animal development. Nature reviews Molecular cell biology. 2008;9(3):219-30.

25. Zhang T, Lv C, Li L, Chen S, Liu S, Wang C, et al. Plasma miR-126 is a potential biomarker for early prediction of type 2 diabetes mellitus in susceptible individuals. BioMed research international. 2013;2013.

26. Jahromi SSM, Jamshidi MM, Farazmand A, Aghazadeh Z, Yousefi M, Mirshafiey A. Pharmacological effects of $\beta$-d-mannuronic acid (M2000) on miR-146a, IRAK1, TRAF6 and NF-KB gene expression, as target molecules in inflammatory reactions. Pharmacological Reports. 2017;69(3):479-84. 
27. Guay C, Regazzi R. Circulating microRNAs as novel biomarkers for diabetes mellitus. Nature Reviews Endocrinology. 2013;9(9):513.

28. Fish JE, Santoro MM, Morton SU, Yu S, Yeh R-F, Wythe JD, et al. miR-126 regulates angiogenic signaling and vascular integrity. Developmental cell. 2008;15(2):272-84.

29. Wang S, Aurora AB, Johnson BA, Qi X, McAnally J, Hill JA, et al. The endothelial-specific microRNA miR-126 governs vascular integrity and angiogenesis. Developmental cell. 2008;15(2):261-71.

30. Meng S, Cao J-T, Zhang B, Zhou Q, Shen C-X, Wang C-Q. Downregulation of microRNA-126 in endothelial progenitor cells from diabetes patients, impairs their functional properties, via target gene Spred-1. Journal of molecular and cellular cardiology. 2012;53(1):64-72.

31. Ciccacci C, Di Fusco D, Cacciotti L, Morganti R, D’Amato C, Greco C, et al. MicroRNA genetic variations: association with type 2 diabetes. Acta diabetologica. 2013;50(6):867-72.

32. Herrera BM, Lockstone HE, Taylor JM, Wills QF, Kaisaki PJ, Barrett A, et al. MicroRNA-125a is overexpressed in insulin target tissues in a spontaneous rat model of Type 2 Diabetes. BMC medical genomics. 2009;2(1):54.

33. Parrinello $\mathrm{CM}$, Selvin $\mathrm{E}$. Beyond $\mathrm{HbA} 1 \mathrm{c}$ and glucose: the role of nontraditional glycemic markers in diabetes diagnosis, prognosis, and management. Current diabetes reports. 2014;14(11):548.

\section{Figures}

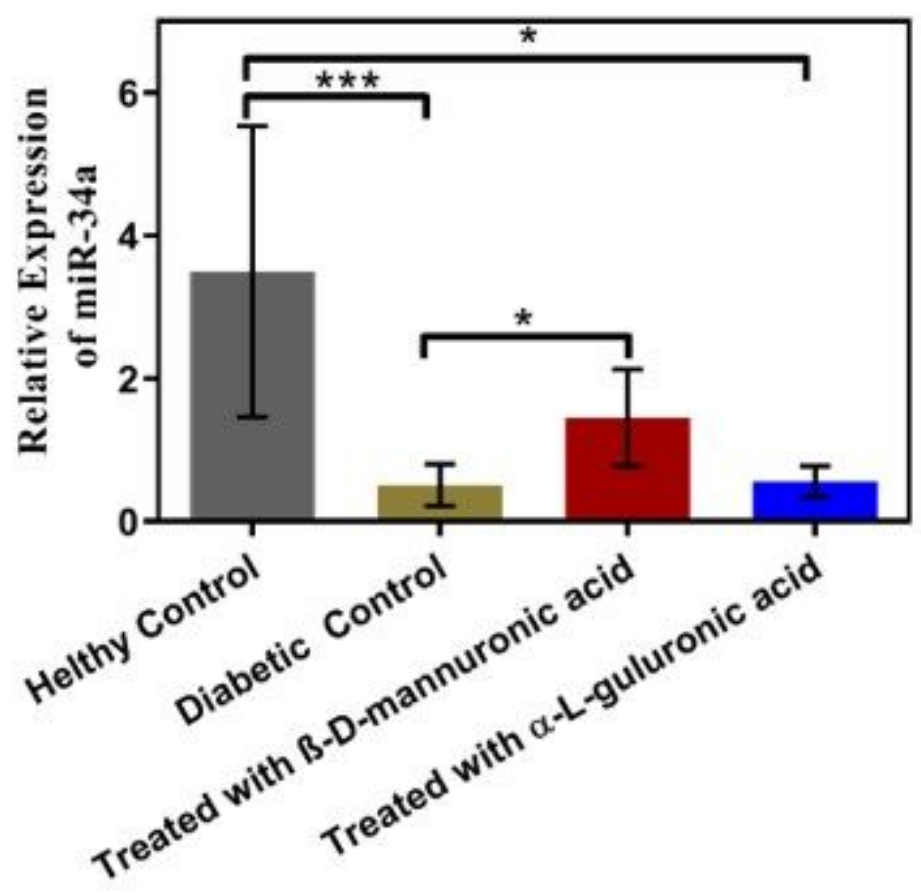


Figure 1

The expression level of miR-34a in sera of healthy control, diabetic control, and treated groups. As shown, treatment with $\beta$-D-mannuronic acid could improve the downregulation of miR-34a expression in diabetic rats. ${ }^{*}, * *,{ }^{* *}$ depict respectively $p \leq 0.05, p \leq 0.01, p \leq 0.001$.

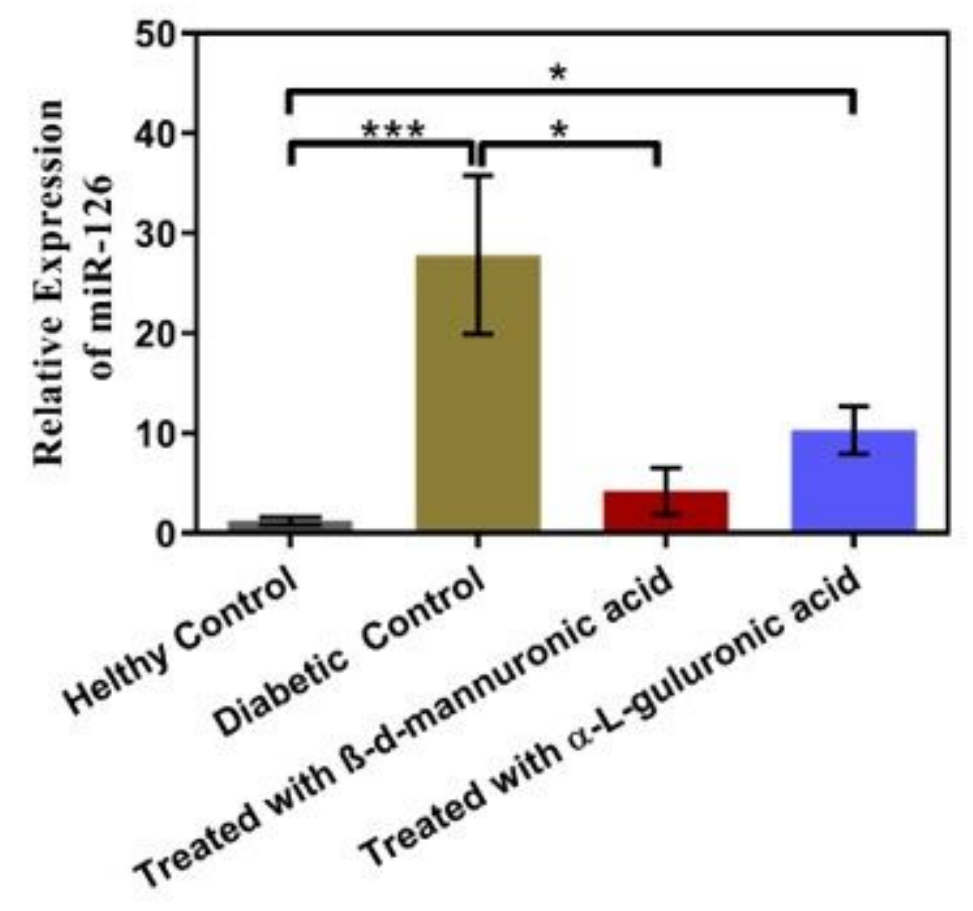

Figure 2

The expression level of miR-126 in sera of healthy control, diabetic control, and treated groups. As shown, treatment with $\beta$-D-mannuronic acid could improve the upregulation of miR-126 expression in diabetic rats. ${ }^{*}, * \star, * \star \star$ depict respectively $p \leq 0.05, p \leq 0.01, p \leq 0.001$. 


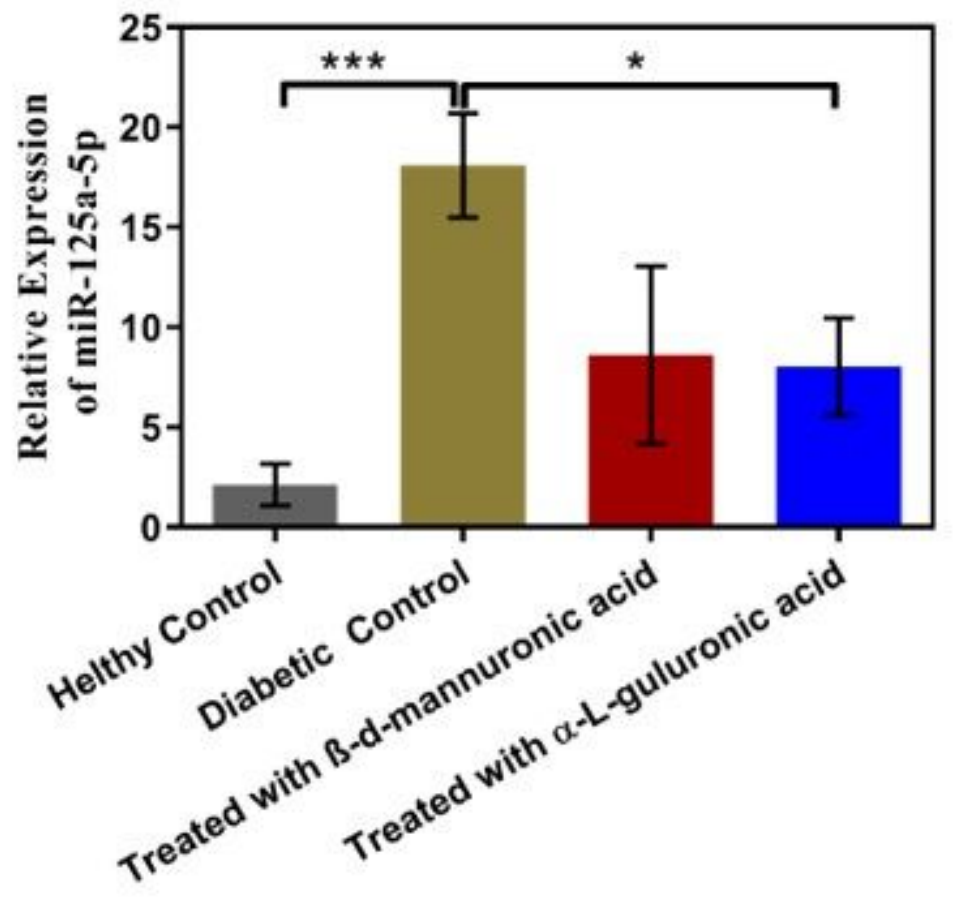

Figure 3

The expression level of miR-125a-5p in sera of healthy control, diabetic control, and treated groups. As shown, treatment with $\beta$-D-mannuronic acid could improve the upregulation of miR-125a-5p expression in diabetic rats. $* * \star, * \star *$ depict respectively $p \leq 0.05, p \leq 0.01, p \leq 0.001$. 